\title{
AN IMPROVEMENT ON GEOMETRY-BASED METHODS FOR GENERATION OF NETWORK PATHS FROM POINTS
}

\author{
Z. Akbari ${ }^{a, *}$ and R. Ali Abbaspour ${ }^{\mathrm{b}}$ \\ ${ }^{a}$ Faculty of Geodesy \& Geomatics Eng., K. N. Toosi University of Technology, Tehran, Iran- akbari.zahra89@gmail.com \\ ${ }^{\mathrm{b}}$ Dept. of Surveying and Geomatics Eng., Engineering College, University of Tehran, Tehran, Iran- abaspour@ut.ac.ir
}

KEY WORDS: Network path, Geometry-based, VGI.

\begin{abstract}
:
Determining network path is important for different purposes such as determination of road traffic, the average speed of vehicles, and other network analysis. One of the required input data is information about network path. Nevertheless, the data collected by the positioning systems often lead to the discrete points. Conversion of these points to the network path have become one of the challenges which different researchers, presents many ways for solving it. This study aims at investigating geometry-based methods to estimate the network paths from the obtained points and improve an existing point to curve method. To this end, some geometry-based methods have been studied and an improved method has been proposed by applying conditions on the best method after describing and illustrating weaknesses of them.
\end{abstract}

\section{INTRODUCTION}

Recently, using the positioning systems such as GPS, which is capable of determining the locations of points, is rapidly increasing. Large volumes of spatial data can be gained using these systems by spending short time and low cost (Song et al., 2010) and (Wang, 2011). Extraction of network paths using low-accuracy data obtained by such systems is one of interest (Kasemsuppakorn et al., 2013). Data obtained from such systems is shown as discrete points, which if connected, can be used in several studies such as in traffic to determine vehicles speed and accessing network path. Error in systems is derivation from centerline of roads and geometry of road networks resulting from cumulating roads centerline causes non-location of points exactly on network lines. Thus, extraction of networks from point set data is very important. Up to now, many studies have been conducted using different geometry-based, mathematical, and statistical methods (Scott, 1994) and (Newson et al., 2009).

Geometry-based methods in roads intersections and high density locations come along with some problems resulting in actual derivation. Aim of this paper is to offer algorithm and conditions to minimize errors resulting from such issues. To this end, initially three geometry-based methods available to matching points on network center line (direct line methods, point to point and point to curve) have been studied and results obtained from using them for some sets of observations are compared to the actual path. In order to assess each one of these methods, number of common line and length of the whole path estimated is compared to the actual path and finally, after investigating issues resulting in error and visual illustration of their weaknesses, an optimized method is offered.

\section{BACKGROUND}

In this section, some methods of extracting network path from observed points have been investigated.

\subsection{Direct Line Method}

In this method, a direct line is drawn between two points (Wentz et al., 2003). This method demonstrates the trajectory in the simplest manner. The shorter the temporal distance between obtained points, the more exact the estimation of trajectory. Figure 1 shows trajectory of a set of observations using direct line method.

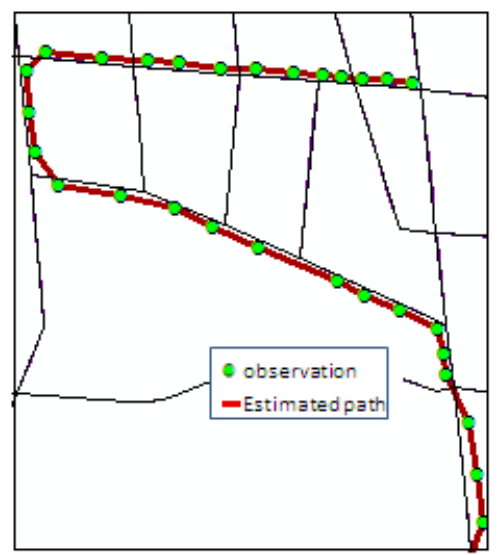

Figure 1. Estimation of the path using direct line method

According to Figure 1, a negligible overlap exists between network lines and estimated path and as noted before, movements is not exactly along middle lines of roads. 


\subsection{Closest Allocation Method}

This method is divided into two groups of point-to-point and point-to-curve.

2.2.1 Point to Point: In this method, each observed point is projected on the closest vertex available on the network (Gkoulalas et al., 2009). The following algorithm defines stages of determining network path in this method:

(In the following algorithm, Vector A contains network points participating in the estimated path, which is void at the beginning).

a) Find the closest point of network $\left(\mathrm{n}_{\mathrm{i}}\right)$ for each of the observed points $\left(\mathrm{O}_{\mathrm{i}}\right)$.

b) Add $n_{i}$ to $\mathbf{A}$, in case $n_{i}$ is not present in $\mathbf{A}$.

c) Repeat these stages for all the observed points.

d) Find the closest path between any two successive points in the vector $\mathbf{A}$.

The total paths obtained show the final path between origin and destination points in this method. Figure 2 shows an example of weaknesses exist in this method.

In such method, there is no guarantee for encompassing all lines of actual path by the estimated path and number of lines in it is always more than the number of lines on the actual path (Timothy et al., 2012). In fact, in very rare, ideal circumstances, this method can provide a satisfactory answer and (Zhu et al., 2006) and (Bernstein et al., 1996). If a network arc were comprised of many vertices, points obtained around that arc would be figured correctly. Contrary, if an arc is only comprised of two vertices at the beginning and the end of a line, the points observed around that will less likely be figured on that, i.e. path divergence will occur (Quddus et al., 2007).

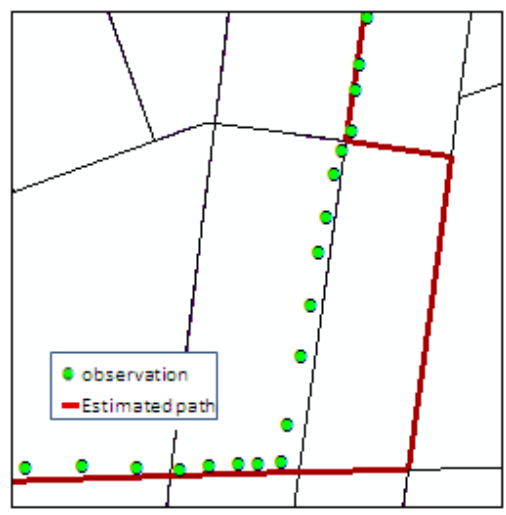

Figure 2. An example of weakness in point-to-point method

2.2.2 Point to curve: In this method, each of the obtained points is mapped on the closest arc of the network. The following algorithm shows the stages of this method: a) Calculate the distance of each Oi from all lines related to each arc

b) (Note: Each arc of the network is consists of several lines)

c) Find the closest line of network arcs (1i) to oi

d) Find base perpendicular to the mentioned line and adding its equivalent points to $\mathrm{A}$

e) Repeat the above points for all observed points

f) Find the closest path between any two successive points in the vector $\mathrm{A}$.

The total paths obtained show the final path between origin and destination points in this method.

Compared to point-to-point method, this method leads to more actual results in the most of the cases. Two obvious advantages of this method compared to point to point method are as follows:

- This method is not limited to vertices and all points located on the network lines attend the beginning of the algorithm.

- Considering that all points of the observed beginning and end are not exactly located on network vertices and keep a distance from them in the point to point method, extra length along network path is to taken into account, through considering perpendicular base in the point to curve method, this extra length is omitted.

Results obtained showed that compared to other methods, point to curve is of more estimation accuracy. However, this method carries drawbacks as well, divided as follows:

- Incorrect estimation in intersections: Probability of error in intersections is high as there is no guarantee that observed points are closest to the correct arc (Fig. 3). This method confronts more problems in intersections compared to point-topoint method (Zhu et al., 2006) and therefore, in a network with more dense lines and networks, which comprise of many intersections, this method is not recommended.

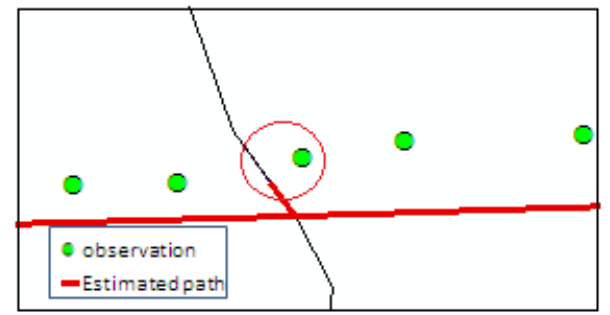

Figure 3. Weakness of point to curve method due to incorrect estimation in intersections

- Ignorance of the lack of connections in intersections: in some cases, due to the lack of network connections in intersections and incorrect dedication, a long path is mistakenly added to the estimated path (see Fig. 4) 


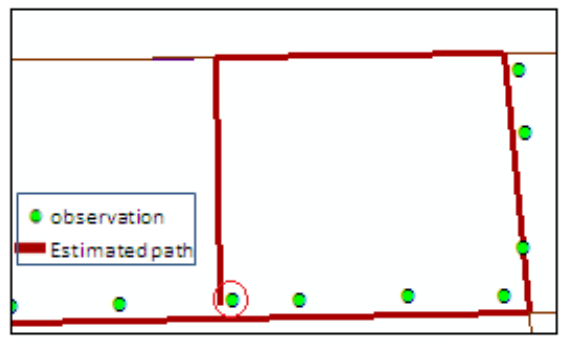

Figure 4. Weakness of point to curve method due to ignoring lack of connection in intersections

- $\quad$ High density of network lines: in networks with lines of higher density, probability of error and incorrect positioning of arcs in the estimated path is higher (Fig. 5). It should be noted that these problem is also seen in point to point method more seriously.

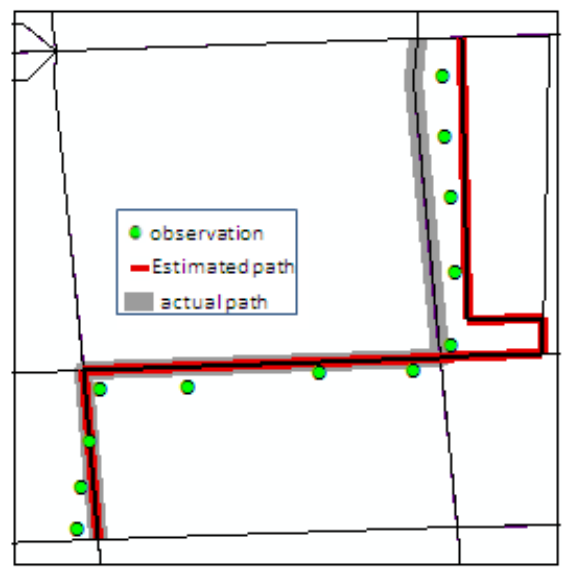

Figure 5. Incorrect path estimation due to high density of network lines

\section{EVALUATION OF EXISTING METHODS}

In order to assess the quality of the method reviewed in the previous sections, three classes of obtained points are modeled using these methods. The number of points and lines common to actual paths was calculated for each case in each method. Results of these evaluations are presented in Table 1 to Table 3.

\begin{tabular}{|c|c|c|c|}
\hline Path name & $\begin{array}{c}\text { Number } \\
\text { of arcs }\end{array}$ & $\begin{array}{c}\text { Number of common } \\
\text { arcs with actual }\end{array}$ & $\begin{array}{c}\text { Length } \\
\text { of path }\end{array}$ \\
\hline Actual Path 1 & 13 & 0 & 1862 \\
Estimated Path 1 & 0 & 0 & 1901 \\
\hline Actual Path 2 & 17 & 0 & 2253 \\
Estimated Path 2 & 0 & 0 & 1436 \\
\hline Actual Path 3 & 10 & 0 & 1488 \\
Estimated Path 3 & 0 & 0 \\
\hline
\end{tabular}

Table 1. Results of evaluation of the direct line method

\begin{tabular}{|c|c|c|c|}
\hline Path name & $\begin{array}{c}\text { Number } \\
\text { of Arcs }\end{array}$ & $\begin{array}{c}\text { Number of common } \\
\text { arcs with actual }\end{array}$ & $\begin{array}{c}\text { Length } \\
\text { of path }\end{array}$ \\
\hline Actual Path 1 & 13 & 11 & 1862 \\
Estimated Path 1 & 17 & 180 \\
\hline Actual Path 2 & 17 & 15 & 2253 \\
Estimated Path 2 & 24 & & 2836 \\
\hline Actual Path 3 & 10 & 9 & 1436 \\
Estimated Path 3 & 12 & 9724 \\
\hline
\end{tabular}

Table 2. Assessing results of point to point method

\begin{tabular}{|c|c|c|c|}
\hline Path name & $\begin{array}{c}\text { Number } \\
\text { of Arcs }\end{array}$ & $\begin{array}{c}\text { Number of common } \\
\text { arcs with actual }\end{array}$ & $\begin{array}{c}\text { Length } \\
\text { of path }\end{array}$ \\
\hline Actual Path 1 & 13 & 13 & 1862 \\
Estimated Path 1 & 15 & 1961 \\
\hline Actual Path 2 & 17 & 16 & 2523 \\
Estimated Path 2 & 20 & & 2680 \\
\hline Actual Path 3 & 10 & 10 & 1426 \\
Estimated Path 3 & 12 & 1658 \\
\hline
\end{tabular}

Table 3. Results obtained from point to curve method

Considering the drawbacks and the results obtained by the evaluation, it is necessary to improve the efficiency of these methods by applying geometrical conditions.

\section{PROPOSED METHODOLOGY}

In order to solve the drawbacks of existing point to curve method (which is shown that is the best geometry-based method), an improvement is proposed in this study.

The following conditions are considered in this method for locating an arc on the path.

a) A buffer around path estimated by direct line method is considered having distance $L$ (Fig. 6). Network lines are considered as a part of final estimated path, only if they are located inside polygon resulting from buffer.

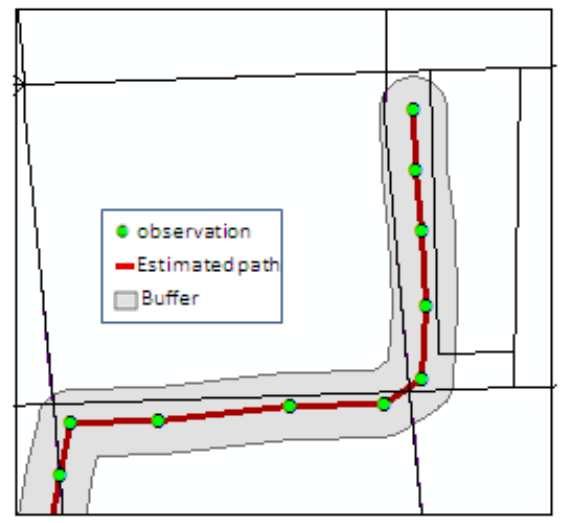

Figure 6. Buffer from estimated path using direct line

This condition is shown as the following algorithm (Fig. 7) and lines available at the shortest path between each two consecutive point are filtered. 
Choosing different values for $\mathrm{L}$, different results are obtained. On the other hand, by increasing or decreasing this L, accuracy of estimated path cannot be verified in the same way. Results obtained from estimation of a path by choosing different values for $\mathrm{L}$ are shown in Table 4. (Total length of actual path is 1560 and its lines number is 12)

\begin{tabular}{|c|c|c|c|}
\hline $\begin{array}{c}\text { Value } \\
\text { for } L\end{array}$ & $\begin{array}{c}\text { Number of } \\
\text { total lines }\end{array}$ & $\begin{array}{c}\text { Number of common } \\
\text { arcs with actual }\end{array}$ & Length \\
\hline 30 & 8 & 8 & 1135 \\
50 & 12 & 12 & 1560 \\
100 & 14 & 10 & 1967 \\
\hline
\end{tabular}

Table 4. Results obtained from different values for $L$

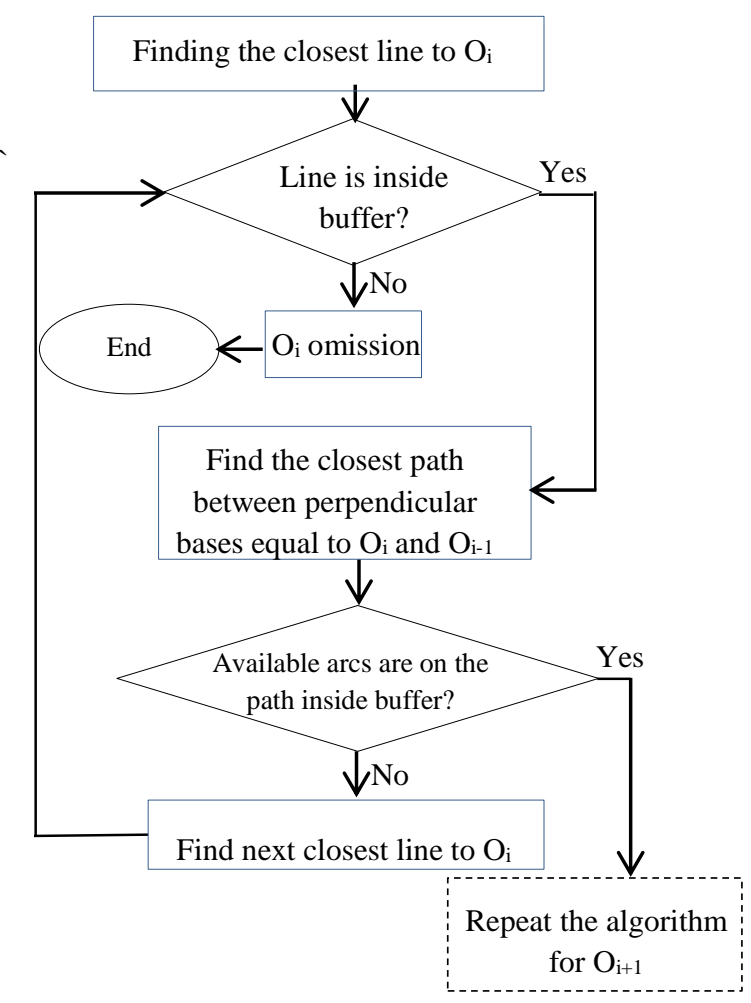

Figure 7. Algorithm for filtering of observed points and lines of the shortest path

In order to choose the best value for $\mathrm{L}$, as length of path estimated by direct line method is much closer to the real length of path (Timothy et al., 2012), length of path can be measured for different values of L. finally it would be possible to choose L, considering length of estimated path, is of less difference to the length estimated by direct line.

b) In the above algorithm, if the closest line between $\mathrm{O}_{\mathrm{i}-1}, \mathrm{O}_{\mathrm{i}+1}$ is on a single arc, then line corresponds to $\mathrm{O}_{\mathrm{i}}$ must be on the same arc.

c) Line or a series of consecutive lines should not be repeated on the final path in reciprocating manner. Such consecutive lines are often results of path derivation from intersection of more than two roads and lack of connections in intersections.

Results of applying the mentioned condition on point to curve method are offered in Table 5.

\begin{tabular}{|c|c|c|c|}
\hline Path name & $\begin{array}{c}\text { Number } \\
\text { of Arcs }\end{array}$ & $\begin{array}{c}\text { Number of common } \\
\text { arcs with actual }\end{array}$ & $\begin{array}{c}\text { Length } \\
\text { of path }\end{array}$ \\
\hline Actual Path 1 & 13 & 13 & 1862 \\
Estimated Path 1 & 13 & 1862 \\
\hline Actual Path 2 & 17 & 16 & 2523 \\
Estimated Path 2 & 19 & & 2545 \\
\hline Actual Path 3 & 10 & 10 & 1426 \\
Estimated Path & 10 & & 1426 \\
\hline
\end{tabular}

Table 5. Assessing results by proposed conditions

It is only in rare cases that are extraordinarily high density of network lines would be a little out of the way.

\section{CONCLUSION}

Determining network path from points is an issue extensively investigated in many studies. Among different methods, this study addressed results obtained from three geometry-based methods. It is evident that such methods along with such setbacks as path derivation in intersections, non-connection of lines in locations and decrease in network accuracy in high-density locations. Available methods for solving such issues are often complicated due to mathematical and statistical base. Therefore, this study uses a fully geometric method to determine network path. Results obtained from these methods showed that point to curve method is more functional. Thus, this method was considered as the basis of study and its setbacks were mitigated through applying conditions and suggested algorithms. Results obtained showed that restricting areas of lines attended the estimated path and applying such conditions as non-existence of arcs in a reciprocating manner along the path, path derivation is minimized.

\section{REFERENCES}

Bernstein, D., Kornhauser, A., 1998. Map Matching for Personal Navigation Assistants, 77th Annual meeting, The Transport Research Board, Jan 11-15.

Gkoulalas-Divanis, A., Verykios, V.S., and Bozanis, P., 2009. A network aware privacy model for online requests in trajectory data. Data and Knowledge Engineering, 68, 431-452.

Kasemsuppakorn, P., Karimi, H.A., 2013. A pedestrian network construction algorithm based on multiple GPS traces. Transportation Research Part C: Emerging technologies, 26, 285-300.

Newson, P., Krumm, J., 2009. Hidden Markov Map Matching Through Noise and Sparseness, ACM SIGSPATIAL Conference on GISystems.

Quddus, M.A., Ochieng, W.Y., Noland, R.B., 2007. Current Map-Matching Algorithms for Transport 
Applications: State-of-the Art and Future Research Directions, Trans. Res. C, 15(5), 312-328.

Scott, C., 1994. Improving GPS Positioning for MotorVehicles through Map Matching. Proc. of ION GPS-94, Seventh International Technical Meeting of the Satellite Division of The institute of Navigation, 1391-1400.

Song, X.F., Raghavan, V., and Yoshida, D., 2010. Matching of vehicle GPS traces with urban road networks. Current Science, 98 (12), pp. 1592-1599.

Timothy, C., Matisziw, a., and Emre, D., 2012. Inferring network paths from point Observations, International Journal of Geographical Information Science, 1979-1996.

Wang, J., 2011. A weighted clustering algorithm for clarifying vehicle GPS, IEEE international geosciences and remote sensing symposium, New York: IEEE, 2949-2952.

Wentz, E.A., Campbell, A.F., and Houston, R., 2003. A comparison of two methods to create tracks of moving objects: linear weighted distance and constrained random walk. International Journal of GIS, 17 (7), pp. 623-645.

Zhou, J., Golledge, R., 2006. A Three-Step General Map Matching Method in the GIS Environment: Travel/Transportation Study Perspective. International Journal of GIS, 8(3), 243-260. 